\title{
Fully Automatic Spot Welding System for Application in Automotive Industry
}

\author{
Peter Puschner ${ }^{1}$, Marcus Klein ${ }^{1}$, Gerhard Kölzer ${ }^{1}$
}

${ }^{1}$ ELMATECH GmbH, 51597, Morsbach, Germany

Received: 29 Sept., 2015

Accepted: 18 Nov., 2015

E-mails: p.puschner@elmatech.de (PP), m.klein@elmatech.de (MK), g.koelzer@elmatech.de (GK)
This is an Open Access article distributed under the terms of the Creative Commons Attribution Non-Commercial License which permits unrestricted non-commercial use, distribution, and reproduction in any medium provided the original work is properly cited.
Abstract: A Virtual Machine has led to a fully automatic spot welding system. All necessary parameters are created by measuring systems and algorithms running in the Virtual Machine. A hybrid operating circuit allows the Virtual Machine to read the exact process voltage between the tips of the electrodes every $50 \mu \mathrm{s}$. Actual welding voltage and current allow for the first time reading process impedance, electric power and total energy being transferred to the spot weld. Necessary energy input is calculated by a calorimetric model after measuring the total thickness of the materials to be welded as soon as the welding gun is positioned at the workpiece. A precision potentiometer implemented in the gun delivers the total material thickness within the $0.1 \mathrm{~mm}$ range during the pre-pressure phases. The internal databank of the Virtual Machine controls all essential parameters to guide the total welding process. Special generator characteristics of the welding power unit are created by the Virtual Machine just during the upslope and the welding phases. So the process will be initialized in differentiating the kind of material, mild steel or high strengthen steel. This will affect the kind of energy input and current decrease during the upslope and downslope phases.

Key-words: Automation/Robotic; Control and monitoring in welding; Power sources; Carbon and low alloy steels; Welding metallurgy; High alloy steels; Welding metallurgy; Maintenance (repair) welding.

\section{Introduction}

In decades of years the spot welding process, with which yearly millions of automobile vehicles are produced, was controlled by current and time. The preset welding time and the current level defined the nugget volume and so the result of the weld. With introducing adhesives and a daily growing number of different kind of high strengthen steel new initial conditions for the assembly to be weld are created which makes it more complicate in resolving good welding results. Not only current and time were any longer responsible for the output but more than ever also the welding voltage. The actual voltage to be measured directly between the tips of the electrodes defines together with the actual welding current the process resistance which individually is changing from spot to spot. Because of the loss of measuring cables just to the touching points between the electrode tips and the material to be welded, there was no option known for measuring the exact welding voltage and as a result of this the momentary process resistance.

The energy input - and depending on this nugget diameter, volume and technological values - however is the result of current, process resistance and time during the whole process. The physical background to this is well known for a long time: Energy is created by the integral of current in square multiplied with the impedance over time $\left(e=\int i(t)^{2} * r(t) * d t\right.$, with e=energy, $\mathrm{i}=$ current, $\mathrm{r}=$ resistance, $\mathrm{t}=$ time) where current and resistance are changing with time, they are time variant parameters. As long as the varying resistance is neither measured nor used in the weld controller a reliable statement with respect to the energy input must fail.

The investigations started in the year 2004 in finding solutions to measure continuously the impedance between the tips of the electrodes and with this the real momentary process resistance with the aim to make energy input and correlated with this the nugget volume reproducible.

The breakthrough finally was reached by using a so called virtual measuring technology based on measuring cables fixed inside the clamp on both arms being transformed to the electrode tips. This new created solution eliminates all disturbing and parasitic side effects existing on the way from the measuring points inside the clamp to the electrode tips [1]. As a result of the investigations which started in close cooperation ten years ago 
with the German OPEL company for creating easy to handle spot welding units for the worldwide repair of vehicles in body shops (Figure 1) and later since the year 2008 by the German VW group this new and evident technology also was made available for automotive production lines (Figure 2).

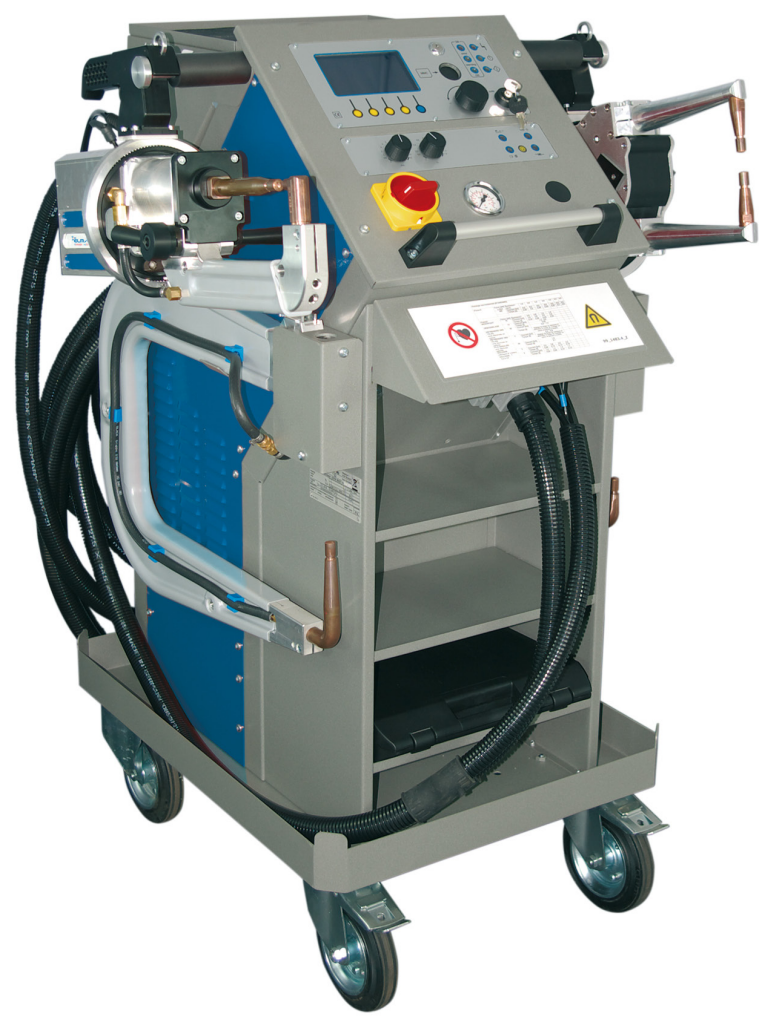

Figure 1. Fully automatic spot welder for body shops, ELMATECH-VISION, C-gun $13 \mathrm{~kg}$, X-gun $12 \mathrm{~kg}, 10 \mathrm{kHz}$ transformer, controlled by Virtual Machine.

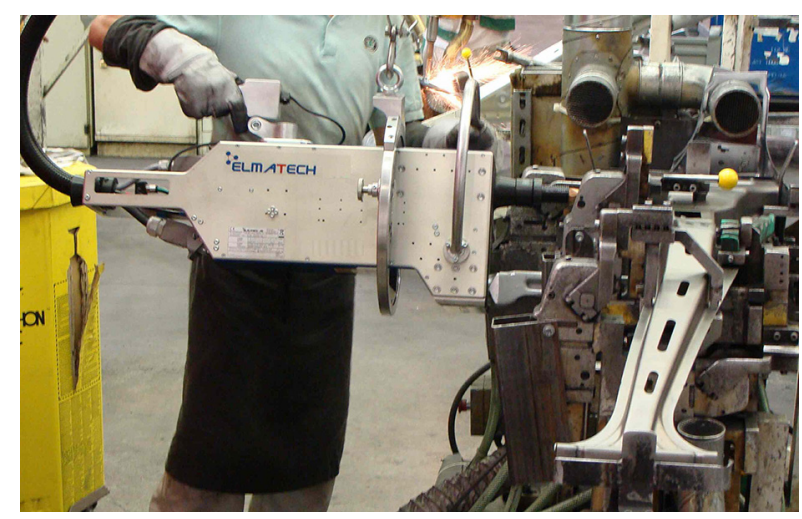

Figure 2. Fully automatic spot welder for automotive production, $10 \mathrm{kHz} \mathrm{C}$-gun (Czechoslovakia).

As a side effect the so developed welding guns based on geometrically small sized $10 \mathrm{kHz}$ transformers and new gun design mainly on aluminum materials there were saved around 60 to 70 percent of the weight in comparison with guns build in state of the art. So the project got the name "Light Weight Gun" with "Intelligent Welding Control” (in German "Leicht-Bau-Zange" LBZ and "Intelligente Schweiß Steuerung” ISS (Figure 3)). 


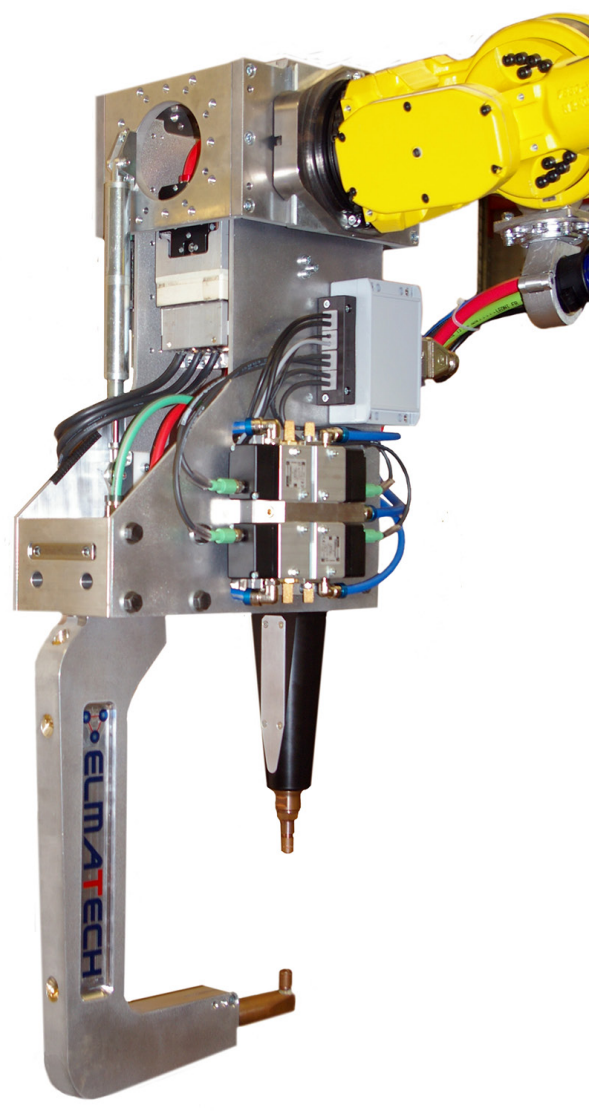

Figure 3. Fully automatic robot welding C-gun with $10 \mathrm{kHz}$ transformer, weight $48 \mathrm{~kg}$.

\section{Fully Automatic Process Control in Robot Application, No Settings Required}

As soon as the robot has positioned the welding gun, the start signal from the robot will be received from the control unit, which is using a Virtual Machine (VM) based on microcomputer design. The control unit first closes the gun by activating the main cylinder by the servo pneumatic system and at the same time balances the gun by a fully automatic operating floating system also being supervised by servo pneumatics within milliseconds. So the gun is floating not depending on the space angle under which it will operate depending on the robot positioning program. As soon as the electrodes create force to the workpiece the overall thickness of the sheets to be welded is measured with an accuracy of $+/-0.1 \mathrm{~mm}$.

The overall thickness leads to a parameter selection from the internal data bank of the VM (Figure 4), with these parameters the total process will be guided. It is an essential attribute of energy control that during the starting phases the process resistance will be treated with care (no explosions and splatters) so the current can do its heating work mainly on the contact points between the different metal sheets and create temperature to form a nugget starting at the points where needed (Figure 5: "Intelligent Welding Control" (in German "Intelligente Schweiß Steuerung" ISS). Thousands of weld trials showed in an explicit way that the result of explosions and splatters lead inevitable to a breakdown of the process resistance to roughly half the value which is followed by constant current driven processes to half power input and after the preset welding time to a loss of energy input of nearly $50 \%$. With the new strategy a promotion effect by the increasing specific resistance of steel leads to optimal temperature increase in the just initialized welding area. Whenever just in the beginning a small weld nugget is created it will be increased by growing energy input and catch all steel plates to generate the wanted output, the weld nugget with the necessary diameter. 
$\mu \mathrm{Ohm}$

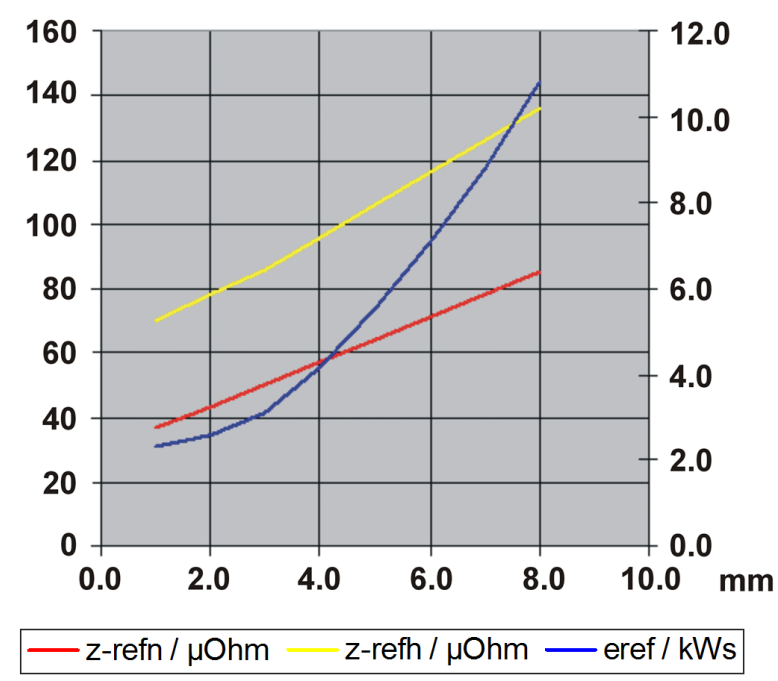

Figure 4. Main Parameters form the synergic table of the VM over thickness ( 0 to $10 \mathrm{~mm}$ ), energy input between the electrode tips (kWs, blue, 0 to $12 \mathrm{kWs),} \mathrm{process} \mathrm{reference} \mathrm{resistance} \mathrm{mild} \mathrm{steel} \mathrm{(} \mu \mathrm{Ohm}$, red, 0 to $120 \mu \mathrm{Ohm}$ ) process reference resistance high strengthen steel ( $\mu \mathrm{Ohm}$, yellow, 0 to $120 \mu \mathrm{Ohm}$ ).

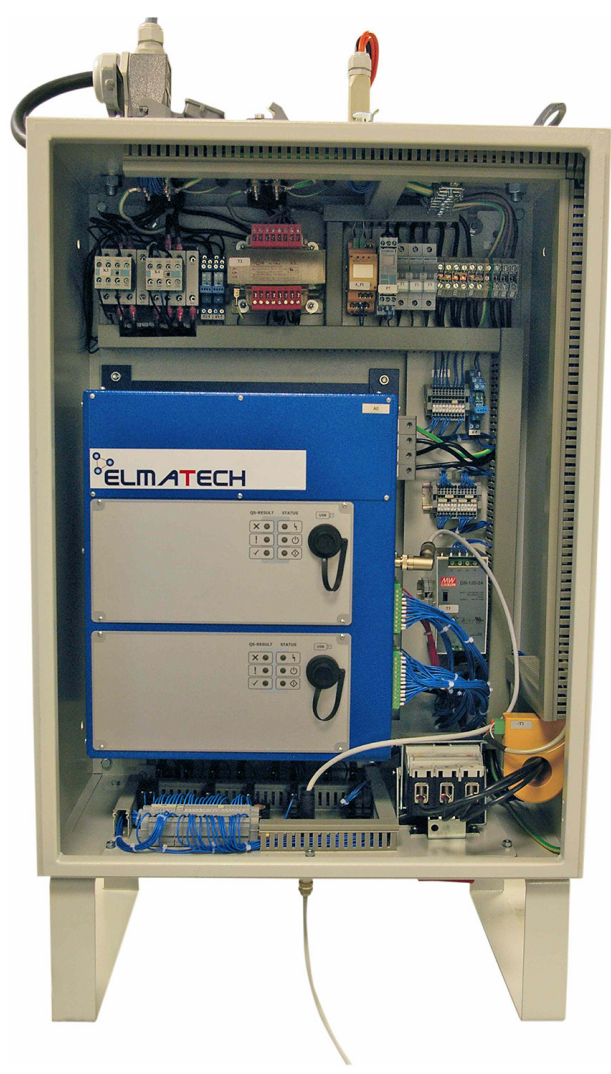

Figure 5. Weld control unit with Virtual Machine.

The point of finalizing the process occurs as soon as the energy input is reached, which was defined by the overall thickness and the corresponding preset energy from the data bank of the VM (Figure 6). 


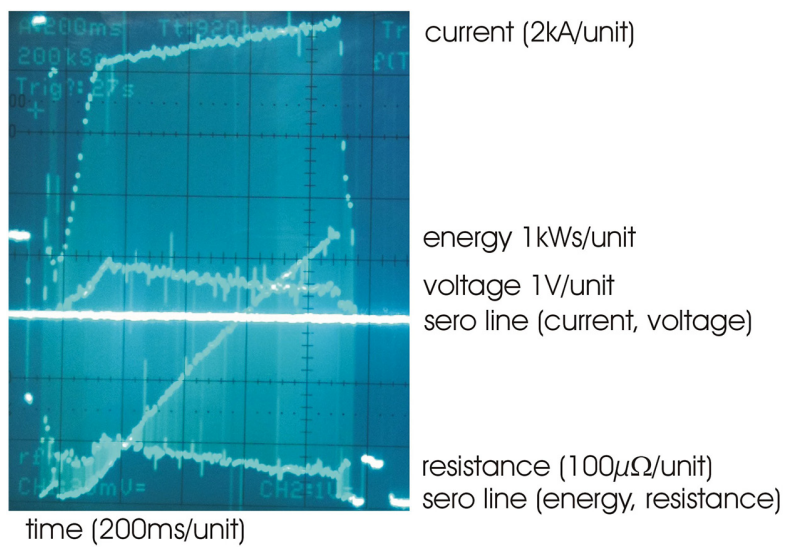

Figure 6. Different parameters during the automatic welding operation. Process is finalized when energy reaches $4.4 \mathrm{kWs}$ as a preset value depending on overall thickness.

During the starting phases the process will be controlled by a virtual generator having variable an programmable static and dynamic characteristics which are realized at the electrode tips for each different process phases. It is the intention of the structure of the Virtual Machine to create different generator characteristics within each cycle of 50 microseconds, which opens a wide field to offer the best and optimal generator behavior for each detectable process phases. That means looking from the electrical side that the output of the trans-former / rectifier is without any losses directly coupled to the sheet surfaces of the workpiece.

Monitoring the electrical parameters just during the starting phases the VM recognizes confidently if there is at least one sheet of high strengthen steel in the combination to be welded. This will cause the VM to influence the speed of energy input just during the starting phases and the decrease at the final phases. Explosions and splatters in the starting and welding phases as well as coarse grid mainly depending on the final phases are suppressed as well.

\section{Simple Robot Interface, Handshake Between Robot and VM Control}

Up to now the robot is guiding main and balance cylinders, the weld current is controlled by the weld control unit. For that purpose the different systems must have been cross-linked with the robot control. This kind of cross-linking is not applicable when using the Elmatech control with the inside operating Virtual Machine with fully automatic operating the welding process and the gun including the balance system.

The control with a Virtual Machine completely takes over the functional control of all gun components, which includes measurement of overall thickness of the sheets, the power control of the welding process and with this providing the static and dynamic characteristic of the electrical generator, the position and force control of the main and the balance cylinder. All pneumatic systems are operating in a servo control mode with high accuracy. This allows the main cylinder to realize exact positioning of the moving electrode before and after the weld in time as short as possible. The main cylinder does not have any mechanics for the prestroke, it is constructed as simple as possible with high reliability using a precision potentiometer for the position feedback.

A full cycle for one spot weld controlled by the VM is described as follows:

1. Start signal form robot to VM (gun is in the correct position at the workpiece)

2. Main cylinder is activated to move the electrode close to the workpiece

3. VM activates the correct gun floating

4. Prepressing by force control

5. Measuring overall sheet thickness

6. Calculating all process parameters by VM 
7. Process start with identifying generator characteristic to determine kind of steel

8. Creating nugget an reaching preset welding current

9. Switching generator characteristic to constant current mode

10. Monitoring energy input for finalizing weld phases

11. Postheating depending on the detected kind of material

12. Postpressing followed by changing servo system from force to position control

13. Open gun to 7th axis position (preset opening position from the robot)

14. Disabling gun floating

15. Signal setting WeldComplete to the robot (handshake)

\section{Physical Basics of the Elmatech Control - Precise Electronic and Mechanic Measurement Technology}

The determination of the overall thickness of the sheets to be welded is performed by a high precision potentiometer integrated in the gun's construction, which is designed for more than 25 million of moving cycles. The VM integrated in the control unit needs only milliseconds to read plausible the overall thickness at the end of the preforce phases. Special measuring cycles and reference cycles create satisfying measurement accuracy within $+/-0.1 \mathrm{~mm}$ as a result [2].

After each tip dressing mode the gun will be referenced using a so called Check Mode to calibrate the gun electrically and mechanically. The Check Mode will last less than 2 seconds and will be done before starting the welding operation outside the workpiece area and only when a tip dressing was performed. The recognized mechanical (distance sero) and electrical parameters will be treated in a mathematical matrix procedure to create virtual measuring points at the electrode tips. Because of these operations every $50 \mu$ s the actual process resistance is disposable and will be used for the energy calculation during the running process.

Main cylinder and balancing cylinder are operating as double acting systems, each of them will be guided by a pair of precise operating servo pneumatic valves. The differential pressure of both cylinder chambers determines with respect to the effective operating plunger the force being created by the cylinder. Force and position regulators operating in the VM are controlling the main and balancing cylinders via servo valves. In a very simple way with this the so called 7th axis of the robot will be managed, which allows the robot to keep control on the opening position after each weld operation. A small opening in the case the robot only has to move aside with no collision possibility this will save time within the total production cycle.

But nevertheless a conventional opening position of 40 or $50 \mathrm{~mm}$ can be realized without data from the robot for a variable opening position. In both cases the VM control unit creates a feedback to the robot that the wanted opening position is achieved and the robot with this handshake can start the moving operation to the next spot position by the signal WeldComplete.

Close to the clamping jaws of the gun's arms there are provided measuring contacts which lead the voltage being measured exactly at those positions to the VM control unit. With the help of the check parameters after the tip dressing operation the unit is able to transfer these measuring points to the electrode tips and to calculate the momentary process resistance every 50 microseconds. This operation simulates measuring cables which are directly in contact with the surface of the sheets to be welded. So this kind of operation creates virtual measuring lines just at these points. The process voltage and the process current (welding current, easily to measure) allow calculating the momentary electrical power and energy input to the spot weld 20,000 times a second. This will guarantee that each spot nugget will receive exactly the needful energy with respect to the measured overall thickness of the sheets. With that the nugget volume and the technological values depending on the energy are achieved.

The virtual measuring lines, which give access to the second electrical parameter voltage at the electrode tips, also open the possibility to transform the characteristics of the power source (inverter-transformer-rectifier) directly to the electrode tips. The result is an operation as if the generator is coupled without any losses and 
inductance straight to the electrodes tips. With that kind of technology the characteristic behavior is adapted to the momentary process status every 50 microseconds. This leads to a better process guiding with less explosions and splatters with the result of perfect welds (Figures 7 and 8 ).

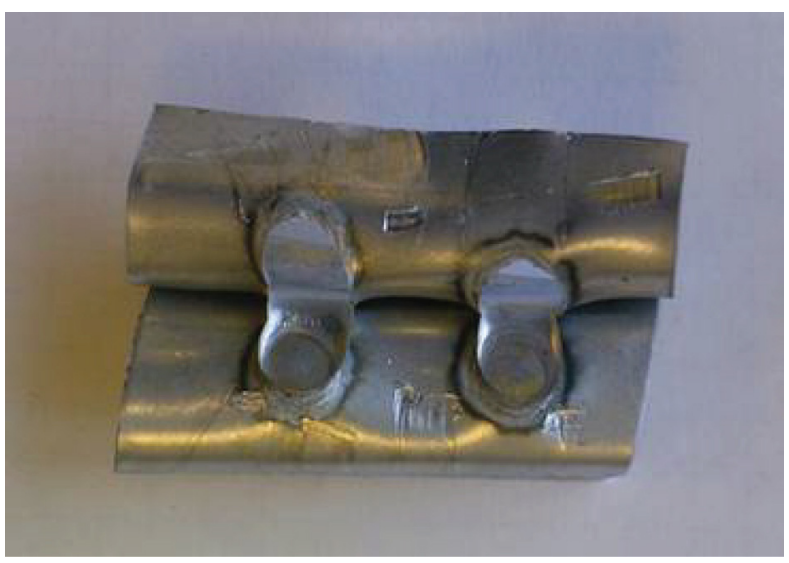

Figure 7. Fully automatic welding of mild steel plates, $0.7 \mathrm{~mm} / 0.7 \mathrm{~mm}$.

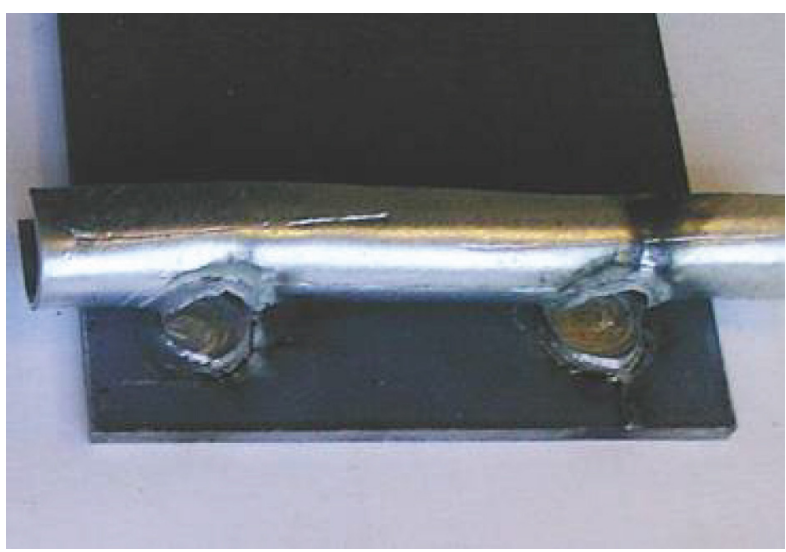

Figure 8. Fully automatic welding of combined mild steel and high strengthen steel plates, $0.7 \mathrm{~mm} / 2.0 \mathrm{~mm}$ (Usibor).

\section{Industrial Applications}

The structure of the technology possibilities when using the synergetic operating Virtual Machine for controlling the spot welding process provided evidence with thousands of well operating spot welders in body shops around the world guaranteeing faultless result in repair situations without any preset parameters of the machine. Continuous tests in technology institutions made sure that the described calorimetric control strategy is correct and leads to strong improved spot welding processes.

The conception of the described technology was taken when building up in first step guns to be used in manual operation in the automotive production for years in order to adapt handling and specific performance of the guns to be fully accepted by the workers as described before. In a second step with the specifications of big automotive players like VW group and

Opel (General Motors) the robot guns as C-type and V-type (similar to X-type) were developed with the aim to have robot guns with low weight operating in a fully automatic mode (Figure 9). The output besides high quality welds is a saving of energy resources by using robots with lower payload which by the way can quicker react as a result of lower masses. 


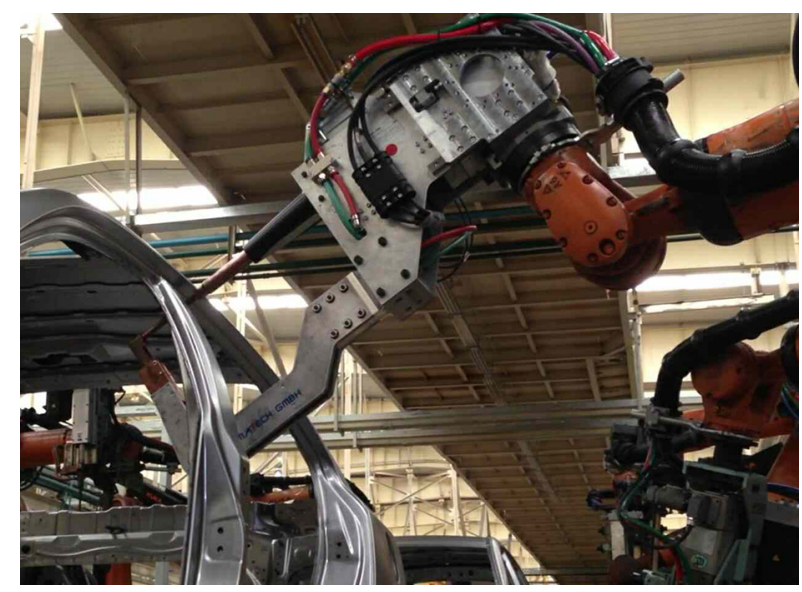

Figure 9. Application automotive industry (China).

\section{Conclusion}

The advantages are on the one side better process results and on the other side time saving when setting a production line in operation. As soon as the robots are taught immediately continuous production can be started. There are no parameter findings or parameter settings for each of thousands spots for one car body necessary, which will take normally a lot of weeks to manage this.

\section{References}

[1] Puschner, P. Einfach nur schweißen. kfz-Betrieb. 2008; 30:1-8.

[2] Puschner, P., Kölzer, G., Geisler, F. Neuartige Schweißsystem für eine flexible Fertigung. Tagungsband 21. DVS-Sondertagung „Widerstandsschweißen 2010“; 2010 May 05-06; Duisburg, Germany. 2010. p. 1-8. 